\title{
Research of Grid-connected PV Inverter with Improved MPPT Algorithm
}

\section{Zhang Haining $^{1}$, Li Qiang ${ }^{2}$, Liu Weiliang ${ }^{2, a^{*}}$, Ma Liangyu ${ }^{2}$,Wang Yinsong ${ }^{2}$}

${ }^{1}$ Key Laboratory of Grid-Connected Photovoltaic Technology, Electric Power Research Institute of Qinghai Power Grid Corporation, Xining, Qinghai, 810008 China

${ }^{2}$ Department of Automation, North China Electric Power University, Baoding, Hebei,071003 China

aemail:Iwlfengzhiying@163.com

Key words: Photovoltaic cells; Boost circuit; Grid-connected inverter; Power feed-forward Abstract: In order to obtain more energy from photovoltaic cells during the inverse process, maximum power point tracking (MPPT) method is adopted in application. Common MPPT methods may cause misjudgment phenomenon, which not only reduce efficiency of PV energy conversion but also may cause grid-connected current distortion. To solve these problems, this paper presents an improved MPPT method and builds a two-level grid-connected inverter system based on MATLAB platform. Simulation result shows that the improved MPPT method could effectively obtain more energy from of photovoltaic cells, and improve the waveform quality of the grid-connected current.

\section{Introduction}

Photovoltaic power generation has increasingly become one of the most promising power generation technologies. The PV array has a strong non-linear characteristics, its output is directly affected by the light intensity, temperature, load and other factors. Therefore, the maximum power point tracking (MPPT) of the solar cell output is particularly important.

In this paper, an improved algorithm is presented, and a grid-connected PV inverter system with current loop control is established. In order to make the given value of the current loop contain the information of the input power, the output power of photovoltaic cells is directly added to the current loop as the feed forward. Simulation model is set up using the Matlab/Simulink platform, and the experiment results show that the above mentioned control method could effectively improve the dynamic response speed of output current of the grid-connected PV system when the environment changes.

\section{Characteristics of Photovoltaic cells}

Under normal working conditions, the output power characteristic curve of the photovoltaic cell has single peak, which is the maximum power point (MPP). Curves of I-U and P-U characteristics when light intensity changes are shown in Fig.1 and Fig.2 respectively. Obviously, output power of photovoltaic cells is mainly influenced by the light intensity. In addition, output power is also influenced by environmental temperature[1,2].

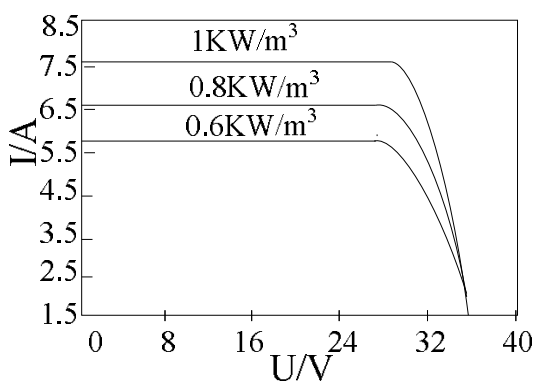

Fig.1 I-U characteristic with different light intensity

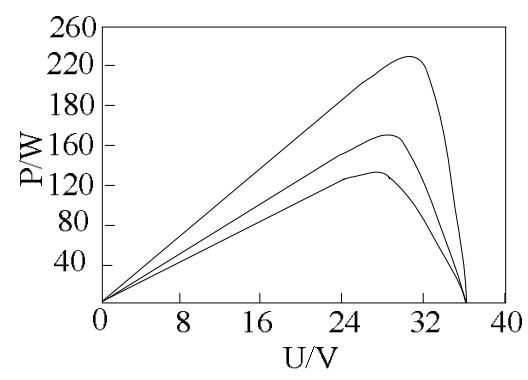

Fig.2 P-U characteristics with different light intensity 


\section{Structure of the MPPT circuit}

In this paper, boost circuit is adopted for the MPPT, as shown in Figure 3.

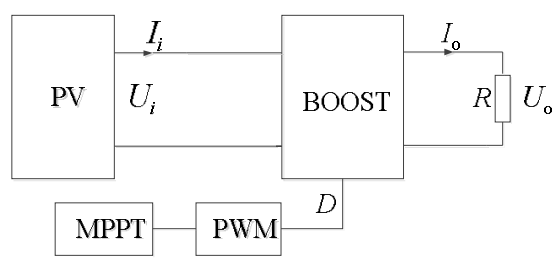

Fig.3 Structure of the MPPT circuit

The relationship between input and output voltage of Boost circuit is:

$$
U_{\mathrm{o}}=U_{i} \frac{1}{1-D}
$$

according to the power balance, there is:

$$
U_{i} I_{i}=U_{\mathrm{o}} I_{\mathrm{o}}
$$

for purely resistive load, the power consumption can be expressed as:

$$
U_{i} I_{i}=\frac{U_{i}^{2}}{(1-\mathrm{D})^{2} R}
$$

The equivalent resistance is:

$$
\mathrm{R}_{\mathrm{e} q}=\frac{U_{i}}{I_{i}}=(1-D)^{2} R
$$

This shows, when $\mathrm{R}$ is fixed, the greater the duty ratio $\mathrm{D}$ is, the smaller the equivalent resistance is. Relationship between the output power $\mathrm{P}$ and the duty ratio D is shown in Fig.4.

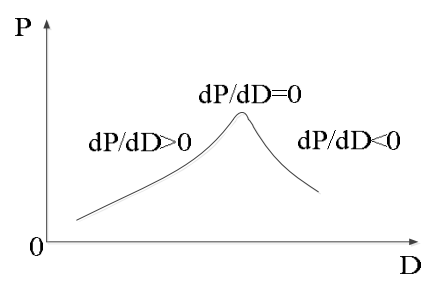

Fig.4 Relationship between the output power $\mathrm{P}$ and the duty ratio D

\section{Disturbance observation method and its improvement}

Disturbance observation method is the most commonly used MPPT method [3-5]. The basic principle is: First, disturb the output voltage of the photovoltaic cell, and observe output power changes of photovoltaic cells. Secondly, decide the next time's disturbing direction of output voltage according to the changing trends of output power so that the photovoltaic cell to work at the maximum power point eventually. For fixed-step disturbance observation method, if the disturbance step is too large, the oscillation problem can not be avoided; if the disturbance step is too small, the tracking speed is quite slow. Based on the output characteristic curve of photovoltaic cells, this paper proposes an improved perturbation observation method.

\section{Principle of improved disturbance observation}

As shown in Fig.4, when the working point is far away from the maximum power point, the $\left|\frac{\mathrm{dP}}{d D}\right|$ is big; when the working point is close to the maximum power point, the $\left|\frac{\mathrm{dP}}{d D}\right|$ is nearly be zero. Considering this rule, the disturbance step could be set as $\Delta U(\mathrm{~K}+1)=\partial\left|\frac{\mathrm{dP}}{\Delta U(\mathrm{~K})}\right|$, where $\partial$ is a constant. This means that in order to solve the contradiction between rapidity and stability of the 
conventional disturbance observation method, the duty cycle variation is automatically adjusted according to the power changing. The flowchart of improved disturbance observation method is shown in Figure 5.

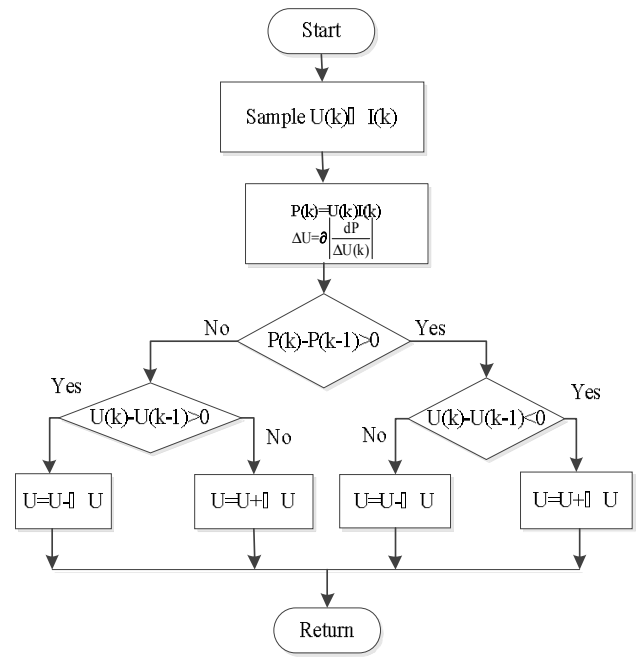

Fig.5: Flow chart of improved perturbation observation method

\section{Comparison of the traditional perturbation observation method and the improved method}

In order to prove the superiority of the presented method, control model of the tradition disturbance observation and the improved disturbance observation is established with Matlab/Simulink ToolBox [6,7]. Set the initial conditions for the standard illumination $1000 \mathrm{~W} / \mathrm{m}^{2}$ and temperature $25^{\circ} \mathrm{C}$. Assumed that the light intensity changes from the $1000 \mathrm{~W} / \mathrm{m}^{2}$ to $800 \mathrm{~W} / \mathrm{m}^{2}$ at the $0.05 \mathrm{~s}$. The simulation results are shown in Fig. 6 and Fig.7.

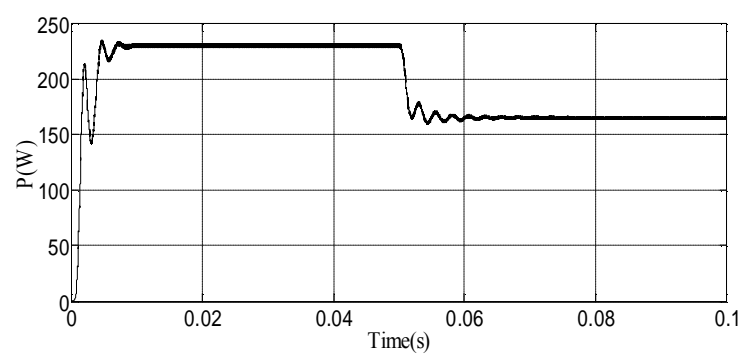

Fig. 6 traditional perturbation observation method

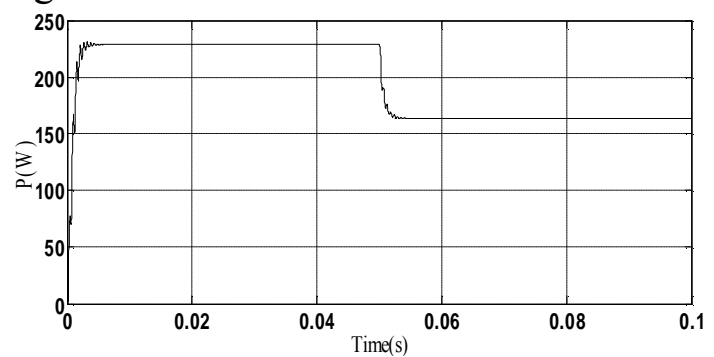

Fig.7 improved perturbation observation method

Assumed that the temperature changes from $25^{\circ} \mathrm{C}$ to $40^{\circ} \mathrm{C}$ in the $0.05 \mathrm{~s}$. The simulation results are shown in Fig.8 and Fig.9.

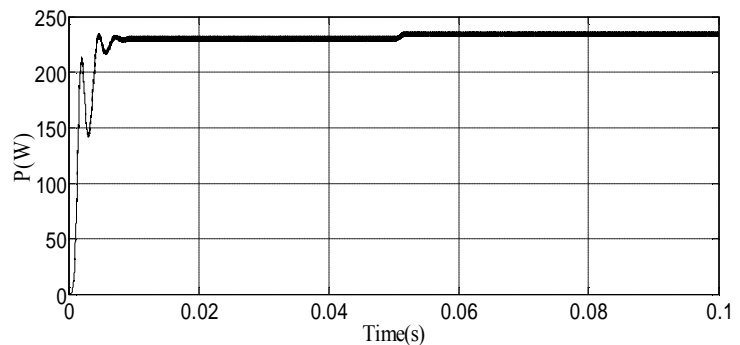

Fig.8 traditional perturbation observation method

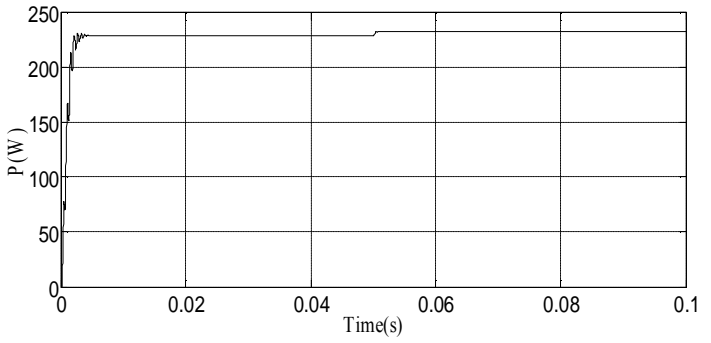

Fig.9 improved perturbation observation method

\section{Model and principle of photovoltaic grid-connected system}

The structure of grid-connected PV inverter system includes DC-DC converter (Boost circuit), single phase full bridge inverter circuit, as shown in Fig.10. The former Boost circuit mainly controls 
the output voltage of PV array and it's function is to realize the maximum power tracking (MPPT) and guarantee the normal work of the level of full bridge inverter circuit; The posterior DC/AC full bridge inverter circuit is to realize the function of inverse, and to achieve the operation of unity power factor of the grid-connected current.

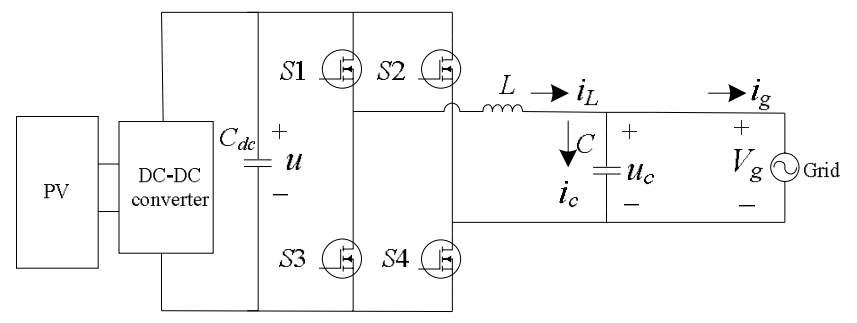

Fig.10 topology of grid-connected PV inverter system

This paper takes a grid-connected current control with power feed-forward to overcome the shortcomings of the common DC voltage control method and eliminate the influence of the grid voltage disturbance to the system. The control strategy of the system block diagram is shown in Fig.11, the current loop realizes the tracking control of AC side current, the given reference of sine wave current $\mathrm{I}_{\text {ref }}$ subtracts the actual grid current $\mathrm{I}$, trigger pulse of grid-connected inverter is obtained by PI regulator compared with carrier, which drives the whole bridge inverter to track AC side current.

Balancing pre-stage output power of photovoltaic cells with the output power of the inverter is the prerequisite of stable work conditions of single phase photovoltaic inverter system. Therefore the reference value of grid-connected current is achieved by photovoltaic cells output power divided by power grid voltage, which multiplies by the phase lock loop PLL voltage phase information as the given current loop. Using power feed-forward link can build direct connection of the input power and output current, when external environment changes, the changing information of photovoltaic cells output power quickly passes to the grid current control link, which improves the dynamic response performance of the single-phase photovoltaic grid inverter systems to the changes of photovoltaic cells output power.

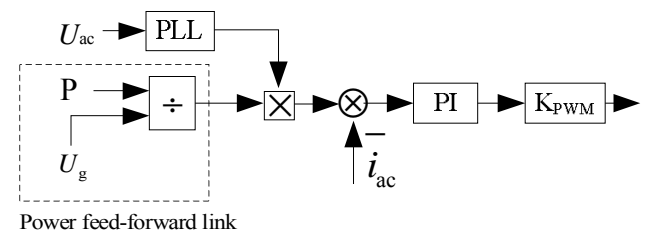

Fig.11 The system block diagram of control strategy

\section{Analysis of simulation results}

Based on the above analysis, simulation model of MPPT method and grid-connected current control method based on the output power compensation is set up using Matlab/simulink ToolBox. For convenience of comparison, the output current value is magnified 10 times. FFT analysis is carried out on the grid-connected current, the simulation results are shown in Fig.12. Fig.12 (a) is the simulation waveform of the fixed step size perturbation method, Fig.12 (b) is the simulation results of the variable step perturbation method. In Fig. 12 (a), grid-connected current harmonic THD $=2.01 \%$; in Fig.12(b), grid-connected current harmonic $\mathrm{THD}=1.98 \%$, which shows the control method presented in this paper meet the requirements of the grid-connected PV inverter. 

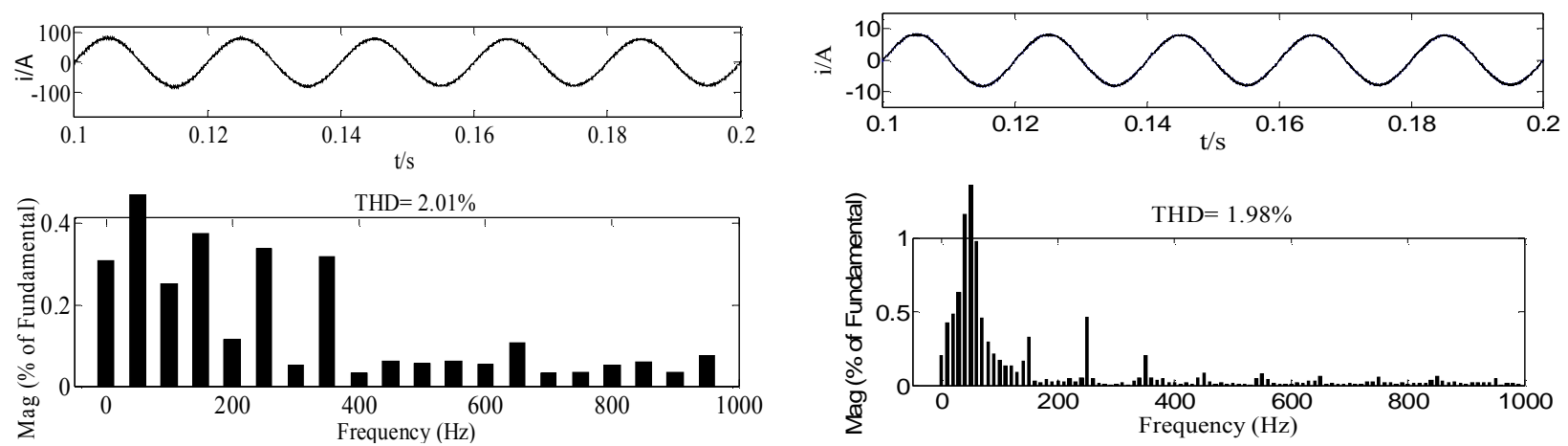

(a)

(b)

Fig.12 FFT analysis of the grid-connected current

In order to verify the response of the grid-connected PV system with the external environment changes, it's assumed that the light intensity changes from $1000 \mathrm{~W} / \mathrm{m}^{2}$ to $600 \mathrm{~W} / \mathrm{m}^{2}$ at the time of 0.2 $\mathrm{s}$, the simulation results are shown in Fig.13. It is known that simulation control model established in this paper can still has good tracking performance, the frequency and phase of grid-connected current is the same as the grid voltage.

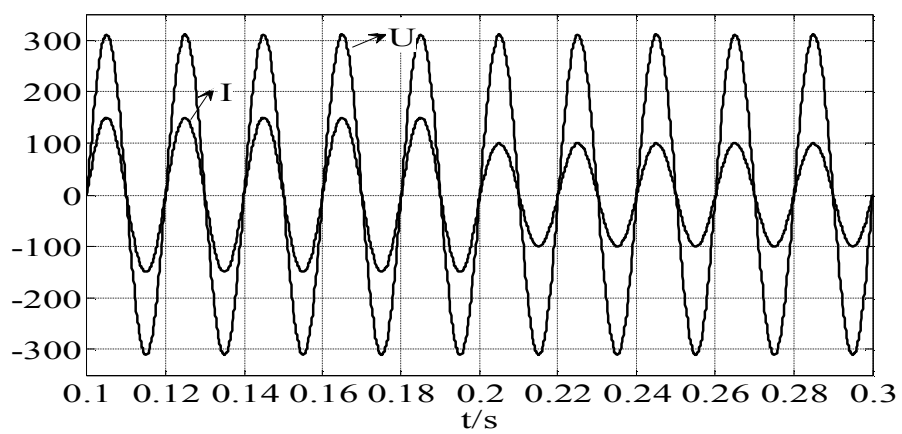

Fig.13 the waveform of grid voltage and grid-connected current

\section{Summary}

In this paper, an improved disturbance observation method is presented, and a single-phase PV grid-connected system is established which adopts the grid-connected control method that combines closed-loop control with power feed-forward. The simulation results shown that presented single-phase PV grid-connected system can accurately track the maximum power point, and improve the waveform quality of the grid-connected current.

\section{Acknowledgment}

The work of this paper is supported by Science and Technology Project of State Grid Corporation of China (2014-Z-Y34A) and "the Fundamental Research Funds for the Central Universities" of North China Electric Power University (2015ZD17).

\section{References}

[1] Zhong Jie. Research of grid connected photovoltaic inverter MPPT and double closed loop control technology [D]. Southwest Jiao Tong University, 2013

[2] Xu Pengwei, Liu Fei, Liu Bangyin, Duan shan-xu. Comparative analysis and improvement of several MPPT methods for PV system [J]. Power Electronic Technology . 05(2007), p. 3-5

[3] Luo Fang, Xu Pengwei, Kang Yong, Duan ShanXu, Mao Cheng-xiong. A photovoltaic system variable step size MPPT Strategy [J]. Telecom Power Technologies. 02(2007), p. 1-5 
[4] Jiao Yang, Song Qiang, Liu Wenhua. Control strategy of photovoltaic grid connected system based on improved MPPT algorithm [J]. Electric Power Automation Equipment.12(2010), p. $92-96$

[5] Mao Meiqin, Yu Shijie, Su Jianhui. PV array with MPPT function Matlab generic simulation model [J]. Journal of system simulation. 05(2005), p. 1248-1251

[6] Feng Tao ,Chen Hua. Photovoltaic array MPPT method research based on improved disturbance observation [J]. Power supply technology. 08(2013), p. 1402-1405

[7] Zhang Huanhuan. MATLAB design and simulation of photovoltaic power generation system [J]. Control engineering. S1(2013), p. 26-29 